\title{
An observable entanglement measure for mixed quantum states
}

\author{
Florian Mintert ${ }^{1}$ and Andreas Buchleitner ${ }^{2}$ \\ ${ }^{1}$ Department of Physics, Harvard University, 17 Oxford Street, Cambridge Massachusetts, USA and \\ ${ }^{2}$ Max-Planck-Institut für Physik komplexer Systeme, Nöthnitzerstr. 38, 01187 Dresden, Germany
}

(Dated: November 5, 2018)

\begin{abstract}
We quantify an unknown mixed quantum state's entanglement by suitable, local parity measurements on its two-fold copy. The associated observable qualifies as a generalized entanglement witness.
\end{abstract}

PACS numbers: 03.67.-a, 03.67.Mn, 89.70.+c

\begin{abstract}
Quantum entanglement is arguably the most bizarre and anti-intuitive feature of quantum mechanics, and, furthermore, the key ingredient for an upcoming quantum information technology. It is the cause of quantumnonlocality, leading to 'spooky action on a distance' and the violation of Bell's inequalities, and opens novel means of data encryption and communication, as well as the efficient factorization of large numbers as a crucial prerequisite of breaking cryptographic codes.

Yet, despite its primordial importance, entanglement is hard to grasp: There is so far no observable which allows for the direct measurement inscribed into a given, arbitrary quantum state. Only indirect ways to assess a given quantum state's degree of entanglement are available: Either through state-selective entanglement witnesses, which are auxiliary observables to identify predefined classes of entangled states - i.e., some a priori knowledge on the state to be detected is required, and other classes of states with exactly the same entanglement properties may remain unidentified. Or through quantum tomography - the experimental reconstruction of the full density operator $\varrho$ from the measurement of a complete set of observables, followed by the evaluation of some entanglement measure, which is in general a nonlinear function of $\varrho$. While witnesses, though efficiently implementable, are no reliable tool for all purposes, tomography implies a rapidly growing experimental overhead as the Hilbert space dimension of the composite system under study increases - either through increasing subdimensions of the subsystems, or through an increasing number of these. This rapidly saturates experimental resources.
\end{abstract}

Therefore, alternative strategies [1, 2] are urgently needed, since experiments now succeed to control increasingly large quantum systems, though meet a hard barrier when it comes to measure efficiently and in real time the available amount of entanglement as their central resource. In the present contribution, we describe how few experimental measurements on a two-fold copy $\varrho \otimes \varrho$ of the mixed state to be analysed provide a tight estimate of the entanglement inscribed in $\varrho$, for bipartite systems of arbitrary finite dimension. This defines a new strategy to overcome the above impediments, and also yields a generalized entanglement witness.

We start out with a short reminder of pure state entanglement and the efficient measurement thereof, what will already fix the algebraic structure which we will use in our subsequent generalization for mixed states: The concurrence $c(\Psi)$ of a finite dimensional bipartite pure state $|\Psi\rangle$ can be expressed through the expectation value of the self-adjoint operator $A=4 P_{-} \otimes P_{-}$, with respect to a two-fold copy $|\Psi\rangle \otimes|\Psi\rangle$ of $|\Psi\rangle$ [3, 4, 5]:

$$
c(\Psi)=\sqrt{\langle\Psi|\otimes\langle\Psi|A| \Psi\rangle \otimes| \Psi\rangle} .
$$

$P_{-}$is the projector on the antisymmetric subspace of the two copies of either subsystem. $c(\Psi)$ is directly accessible in laboratory experiments, through a projective measurement of the antisymmetric component of $|\Psi\rangle \otimes|\Psi\rangle$ in either subsystem, as recently demonstrated for twin photons [6. The same recipe applies for higher-dimensional bipartite systems, and for multipartite generalizations of concurrence [5], since the algebraic structure of (1) prevails.

We now want to generalize this measurement prescription for mixed states of bipartite quantum systems. The key difficulty here stems from the abstract definition of mixed state concurrence through the "convex roof" construction, which takes account of the non-uniqueness of the pure state decomposition of an arbitrary density matrix: Consequently, one has to determine the minimum average concurrence $c(\varrho)=\inf \sum_{i} c\left(\phi_{i}\right)$ of all ensembles $\left\{\left|\phi_{i}\right\rangle\right\}$ that describe the density matrix $\varrho$ [7]. This distinguishes nonclassical from classical correlations typical of a statistical mixture. The optimization problem renders the general evaluation of the convex roof a hard mathematical task, and any direct approximation thereof will yield an upper rather than a lower bound of the entanglement of $\varrho$. However, in order to distinguish separable from entangled states, we need a lower bound, which we will derive by generalizing (1), and which we will show to provide tight estimates of mixed state concurrence. In particular, these estimates are experimentally directly accessible, through a small number of projective measurements, for arbitrary $\varrho$.

Let us start from the observation that the two-fold copy $|\Psi\rangle \otimes|\Psi\rangle$ is symmetric with respect to the exchange of the copies of both subsystems. This is the reason why expectation values of $P_{-} \otimes P_{+}$or $P_{+} \otimes P_{-}$, with $P_{+}$the symmetric counterpart of $P_{-}$, do not contribute to pure state concurrence (1), and a finite expectation value of either observables unambiguously characterizes a twofold copy of a state to be mixed. A positive expectation values 
of $P_{-} \otimes P_{-}$on the other hand can be not only due to entanglement of the underlying state, but also due to its mixing.

Combining these two observations, we conjecture that

$$
(c(\varrho))^{2} \geq \operatorname{Tr}\left(\varrho \otimes \varrho V_{i}\right),
$$

$(i=1,2)$ with $V_{1}=4\left(P_{-}-P_{+}\right) \otimes P_{-}$, and $V_{2}=4 P_{-} \otimes$ $\left(P_{-}-P_{+}\right)$. The proof of this inequality is defered to the Appendix below.

Inequality (2) implies the following important consequences:

- The lower bound on its right hand side can be expressed in terms of the purities of $\varrho, \varrho_{r}^{(1)}$, and $\varrho_{r}^{(2)}$,

$$
\operatorname{Tr}\left(\varrho \otimes \varrho V_{i}\right)=2\left(\operatorname{Tr} \varrho^{2}-\operatorname{Tr}\left(\varrho_{r}^{(i)}\right)^{2}\right)
$$

with $\varrho_{r}^{(i)}$ the reduced density matrix of either subsystem, in close analogy to the expression $c^{2}(\Psi)=$ $2\left(1-\operatorname{Tr}\left(\varrho_{r}^{(i)}\right)^{2}\right)$ for pure state concurrence [8].

- There is an interesting interpretation of $V$ : Any non-vanishing contribution to the expectation value on the right hand side of (2), where the mixedness of the state is already substracted, must be due to nonvanishing quantum correlations insribed in $\varrho$ : Since the left hand side, $c(\varrho)$, is strictly positive for entangled states, and vanishes exactly for separable states, $\operatorname{Tr}\left(\varrho \otimes \varrho V_{i}\right)$ must be non-positive for any separable density operator. Conversely, positive values of $\operatorname{Tr}\left(\varrho \otimes \varrho V_{i}\right)$ unambiguously identify $\varrho$ as entangled. Consequently, $V$ qualifies as a generalized entanglement witness, applicable for arbitrary states, without using a priori knowledge on $\varrho$.

- Moreover, and most importantly, our new lower bound is given in terms of expectation values of $P_{-}$and $P_{+}$. Hence it can be directly measured, as the probabilities of finding the two-fold copies of each state's individual subsystems with positive or negative parity. This implies only little overhead as compared to the experimental measurement of pure state concurrence [6].

Let us assess the tightness of (2), by evaluating the expectation value of $V$ for increasingly mixed random states of $2 \times 5$ and $3 \times 3$ dimensional systems. The random states were obtained by random unitary evolution of a pure state of a tripartite system, composed of the bipartite subspace which supports the desired random states, and an environment component, followed by a subsequent trace over the environment, at different times [9]. The estimate (2) is then compared with the states' concurrence in quasi pure approximation (qpa) 9] in Fig. 1. for samples of 600000 random states. Qpa is known to provide very good approximations of a mixed state's concurrence if the mixing is not too large (hence the label "quasipure"). Our new bound is seen to be only slightly weaker than qpa, as clearly demonstrated by the present comparison. Indeed, the comparison is in most cases excellent, in particular for weakly mixed states. Only for a relatively small portion of relatively strongly mixed (and weakly entangled) states does (2) take negative values (while qpa remains positive), and thus provide an inconclusive result.

Thus, Eq. (2) provides a reliable and directly measurable bound for an unknown state's concurrence. How do the necessary experimental resources scale with the system's size (determined by the individual dimensions of the factor spaces)? According to the explicit form of $V$, we have to determine the state's weight on the symmetric and antisymmetric subspaces of the two copies of each of its components. Consequently, for a bipartite state, with $\mathrm{id}_{\mathrm{i}}=P_{-}^{(i)}+P_{+}^{(i)}, i=1,2$, two measurements need to be performed (here we assume that such parity measurement can be performed by measuring one single observable, independently of the subsystems' dimension). This is in favourable contrast to a tomographic measurement, where $d^{4}-1$ observables need to be measured [10, with $d$ the dimension of the subsystems.

Let us conclude with a brief digression on what we understand by a two-fold copy of an unknown quantum state, since this ansatz, while widely accepted [1, 2, 3, 4, still raises some controversy in the literature [11]: What we do assume here is the availability of a reliable source producing two faithful copies of the same state $\varrho$. Thus, the experimentalist who wants to implement our measurement scheme has to be certain about the source providing $\varrho \otimes \varrho$, but can be perfectly ignorant about the initialization of the source, and, hence, of the specific state $\varrho$ which is delivered in a two-fold copy. In itself, the preparation of two identical copies of $\varrho$ is a very realistic experimental task, given the stunning control over the microscopic constituents of matter, e.g., in state of the art quantum optical experiments.

We are indebted to Luiz Davidovich, Paulo Henrique Souto Ribeiro, and Stephen Patrick Walborn for fruitful discussions, comments and remarks. This work was supported by a Feodor-Lynen fellowship of Alexander von Humboldt foundation.

Appendix: For completeness, let us prove inequality (2): we show that the estimate applies for the average concurrence of any pure state decomposition $\varrho=$ $\sum_{i}\left|\phi_{i}\right\rangle\left\langle\phi_{i}\right|:$

$$
\begin{aligned}
\operatorname{Tr}\left(\varrho \otimes \varrho V_{i}\right) & =\sum_{i j}\left\langle\phi_{i}\left|\otimes\left\langle\phi_{j}\left|V_{i}\right| \phi_{i}\right\rangle \otimes\right| \phi_{j}\right\rangle \\
& \leq\left(\sum_{i} \sqrt{\left\langle\phi_{i}\left|\otimes\left\langle\phi_{i}|A| \phi_{i}\right\rangle \otimes\right| \phi_{i}\right\rangle}\right)^{2} \\
& =\sum_{i j} c\left(\phi_{i}\right) c\left(\phi_{j}\right)
\end{aligned}
$$

and thus, in particular, for the decomposition that achieves the minimum average concurrence. It is sufficient to demonstrate the inequality's validity for each 

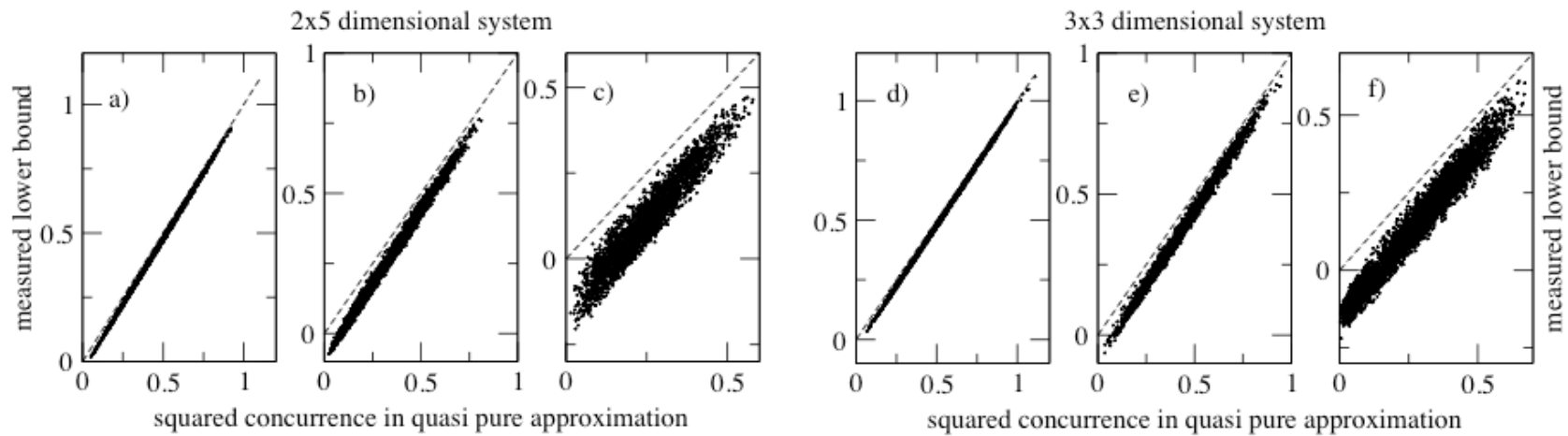

FIG. 1: Squared mixed-state concurrence $c(\varrho)^{2}$, approximated by its measurable lower bound $\operatorname{Tr}\left(\varrho \otimes \varrho\left(V_{1}+V_{2}\right) / 2\right)$ as given by (2), vs. its lower bound in quasi pure approximation, for random states of a $2 \times 5$ dimensional $(a, b$, and $c)$, and of a $3 \times 3$ dimensional system $(d, e, f)$. The different panels represent states with different degrees of mixing: $a$ and $d$ display the case of weakly mixed states $\left(0.2 \leq \sqrt{1-\operatorname{Tr} \varrho^{2}} \leq 0.21\right), b$ and $e$ correspond to the regime of intermediate mixing $\left(0.4 \leq \sqrt{1-\operatorname{Tr} \varrho^{2}} \leq\right.$ $0.405)$, and $c$ and $f$ show strongly mixed states $\left(0.529 \leq \sqrt{1-\operatorname{Tr} \varrho^{2}} \leq 0.533\right)$. The dashed lines indicate equality of both bounds. In particular, for highly entangled states and for states with little mixing the bound is very good; but it also yields a surprisingly good characterization of rather strongly mixed states.

single term in the above sum. For convenience, we rebaptize $\left|\phi_{i}\right\rangle$ as $|\psi\rangle$, and $\left|\phi_{j}\right\rangle$ as $|\phi\rangle$. Given $|\phi\rangle$ 's Schmidt decomposition $|\phi\rangle=\sum_{i} \sqrt{\lambda_{i}}|i\rangle \otimes|i\rangle$ and $|\psi\rangle$ 's expansion in the same one-particle bases, $|\psi\rangle=\sum_{i j} \psi_{i j}|i\rangle \otimes|j\rangle$, (4) can be written as

$$
\begin{aligned}
& \sum_{i \neq j} \psi_{i i}^{*} \psi_{j j} \sqrt{\lambda_{i} \lambda_{j}}-\left|\psi_{i j}\right|^{2} \lambda_{i} \leq \\
& \sqrt{\sum_{i \neq j} \lambda_{i} \lambda_{j}} \sqrt{\sum_{\substack{i \neq j \\
p \neq q}}\left|\psi_{i p} \psi_{j q}-\psi_{i q} \psi_{j p}\right|^{2}},
\end{aligned}
$$

for $V_{2}$, (and analogously for $V_{1}$ ). With the help of the Cauchy-Schwarz inequality, the right hand side (RHS) of this expression can be bounded from below,

$$
\begin{aligned}
\mathrm{RHS} & =\sqrt{\sum_{i \neq j} \lambda_{i} \lambda_{j}} \sqrt{\sum_{\substack{i \neq j \\
p \neq q}}\left|\psi_{i p} \psi_{j q}-\psi_{i q} \psi_{j p}\right|^{2}} \\
& \geq \sqrt{\sum_{i \neq j} \lambda_{i} \lambda_{j}} \sqrt{\sum_{i \neq j}\left|\psi_{i i} \psi_{j j}-\psi_{i j} \psi_{j i}\right|^{2}} \\
& \geq \sum_{i \neq j} \sqrt{\lambda_{i} \lambda_{j}}\left|\psi_{i i} \psi_{j j}-\psi_{i j} \psi_{j i}\right| .
\end{aligned}
$$

For the left hand side (LHS), we find

$$
\begin{aligned}
\text { LHS } & =\sum_{i \neq j} \psi_{i i}^{*} \psi_{j j} \sqrt{\lambda_{i} \lambda_{j}}-\left|\psi_{i j}\right|^{2} \lambda_{i} \\
= & \frac{1}{2} \sum_{i \neq j}\left(\psi_{i i}^{*} \psi_{j j}+\psi_{j j}^{*} \psi_{i i}\right) \sqrt{\lambda_{i} \lambda_{j}}- \\
& \left(\left|\psi_{i j}\right|^{2} \lambda_{i}+\left|\psi_{j i}\right|^{2} \lambda_{j}\right) \\
\leq & \sum_{i \neq j}\left(\left|\psi_{i i} \psi_{j j}\right|-\left|\psi_{j i} \psi_{j i}\right|\right) \sqrt{\lambda_{i} \lambda_{j}} \\
\leq & \sum_{i \neq j}\left|\psi_{i i} \psi_{j j}-\psi_{i j} \psi_{j i}\right| \sqrt{\lambda_{i} \lambda_{j}},
\end{aligned}
$$

where $\left(\left|\psi_{i j}\right| \sqrt{\lambda_{i}}-\left|\psi_{j i}\right| \sqrt{\lambda_{j}}\right)^{2} \geq 0,\left|\psi_{i j}\right|^{2}+\left|\psi_{j i}\right|^{2} \geq$ $2\left|\psi_{i j} \psi_{j i}\right|, \quad \psi_{i i}^{*} \psi_{j j}+\psi_{j j}^{*} \psi_{i i}=2 \Re\left(\psi_{i i}^{*} \psi_{j j}\right) \leq 2\left|\psi_{i i} \psi_{j j}\right|$, and the triangle inequality $\left|\psi_{i i} \psi_{j j}\right|-\left|\psi_{i j} \psi_{j i}\right| \leq$ $\left|\psi_{i i} \psi_{j j}-\psi_{i j} \psi_{j i}\right|$ were used. Thus, LHS $\leq$ RHS, what was our initial claim.
[1] P. Horodecki, Phys. Rev. Lett. 90, 167901 (2003).

[2] F. A. Bovino, G. Castagnoli, A. Ekert, P. Horodecki, C. M. Alves, and A. V. Sergienko, Phys. Rev. Lett. 95, 240407 (2005).

[3] A. Peres and W. K. Wootters, Phys. Rev. Lett. 66, 1119 (1991).

[4] T. A. Brun, Quantum Information and Computation 4, 401 (2004).

[5] F. Mintert, M. Kuś, and A. Buchleitner, Phys. Rev. Lett.
95, 260502 (2005).

[6] S. P. Walborn, P. H. Souto Ribeiro, L. Davidovich, F. Mintert, and A. Buchleitner, Nature 440, 1022 (2006).

[7] C. H. Bennett, D. P. DiVincenzo, J. A. Smolin, and W. K. Wootters, Phys. Rev. A 54, 3824 (1996).

[8] P. Rungta, V. Bužek, C. M. Caves, M. Hillery, and G. J. Milburn, Phys. Rev. A 64, 042315 (2001).

[9] F. Mintert and A. Buchleitner, Phys. Rev. A 72, 012336 (2005). 
[10] M. Nielsen and I. Chuang, Quantum Computation and Quantum Information (Cambridge University Press, Cambridge, 2000).
[11] S. van Enk, quant-ph/0606017 (2006). 